TITLE:

\title{
Nondestructive detection of hidden chemical compounds with laser Compton-scattering gamma rays
}

\section{$\operatorname{AUTHOR(S):~}$}

Hayakawa, Takehito; Ohgaki, Hideaki; Shizuma, Toshiyuki; Hajima, Ryoichi; Kikuzawa, Nobuhiro; Minehara, Eisuke; Kii, Toshiteru; Toyokawa, Hiroyuki

\section{CITATION:}

Hayakawa, Takehito ...[et al]. Nondestructive detection of hidden chemical compounds with laser Compton-scattering gamma rays. Review of Scientific Instruments 2009, 80: 045110.

\section{ISSUE DATE:}

2009-04

URL:

http://hdl.handle.net/2433/85376

RIGHT:

c 2009 American Institute of Physics. 


\title{
Nondestructive detection of hidden chemical compounds with laser Compton-scattering gamma rays
}

\author{
Takehito Hayakawa, ${ }^{1, a)}$ Hideaki Ohgaki, ${ }^{2}$ Toshiyuki Shizuma, ${ }^{1}$ Ryoichi Hajima, ${ }^{3}$ \\ Nobuhiro Kikuzawa, ${ }^{3}$ Eisuke Minehara, ${ }^{3}$ Toshiteru Kiii, ${ }^{2}$ and Hiroyuki Toyokawa ${ }^{4}$ \\ ${ }^{1}$ Advance Photon Research Center, Japan Atomic Energy Agency, Kizugawa, Kyoto 619-0215 Japan \\ ${ }^{2}$ Advanced Energy Generation Division, Institute of Advanced Energy, Kyoto University, Uji, \\ Kyoto 611-0011 Japan \\ ${ }^{3}$ Advance Photon Research Center, Japan Atomic Energy Agency, Tokai, Ibaraki 319-1195 Japan \\ ${ }^{4}$ Research Institute of Instrumentation Frontier, National Institute of Advanced Industrial Science \\ and Technology, Tsukuba, Ibaraki 305-8561 Japan
}

(Received 19 January 2009; accepted 5 April 2009; published online 28 April 2009)

\begin{abstract}
A nondestructive assay method for measuring a shielded chemical compound has been proposed. The chemical compound is measured by using a nuclear resonance fluorescence (NRF) measurement technique with an energy tunable laser Compton-scattering (LCS) $\gamma$-ray source. This method has an advantage that hidden materials can be detected through heavy shields such as iron plates of a thickness of several centimeters. A detection of a chemical compound of melamine, $\mathrm{C}_{3} \mathrm{H}_{6} \mathrm{~N}_{6}$, shielded by 15 -mm-thick iron and 4-mm-thick lead plates is demonstrated. The NRF $\gamma$-rays of ${ }^{12} \mathrm{C}$ and ${ }^{14} \mathrm{~N}$ of the melamine are measured by using the LCS $\gamma$-rays of the energies of up to $5.0 \mathrm{MeV}$. The observed ratio $\left({ }^{12} \mathrm{C} /{ }^{14} \mathrm{~N}\right)_{\exp }=0.39 \pm 0.12$ is consistent with $(\mathrm{C} / \mathrm{N})_{\text {melamine }}=0.5$. (C) 2009 American Institute of Physics. [DOI: 10.1063/1.3125022]
\end{abstract}

\section{INTRODUCTION}

Detection of materials hidden by heavy shields are of importance for many industrial applications, for example, the detection of explosive materials hidden in a package or cargo, and the management of special nuclear materials produced by nuclear power plants. Bremsstrahlung $\gamma$-rays ${ }^{1,2}$ and neutrons ${ }^{3,4}$ have been studied as probes for measuring composition of the shielded materials. Recently, Pruet et al. ${ }^{5}$ has proposed a novel nondestructive detection method of ${ }^{235} \mathrm{U}$ hidden in a cargo transporter by using laser Comptonscattering (LCS) $\gamma$-rays. The LCS $\gamma$-rays are generated by inversed Compton scattering of laser photons with highenergy electrons. The LCS $\gamma$-ray source can generate the energy tunable quasimonochromatic $\gamma$-rays ${ }^{6-9}$ and have been used for studying nuclear physics ${ }^{10,11}$ and industrial applications. ${ }^{12}$ We have proposed an assay method of elemental and isotopic composition of materials hidden by heavy shields by measuring nuclear resonance fluorescence (NRF) scattering $\gamma$-rays with a LCS $\gamma$-ray source (see Fig. 1). ${ }^{14}$ By measuring the NRF scattering $\gamma$-rays, we can detect the nuclear species of interest since the NRF $\gamma$-ray energies depend on the nuclear species, as shown in Fig. 1.

Here we propose an extended nondestructive assay method for measuring molecules or chemical compounds hidden by heavy shields such as iron of thickness of several centimeters. We use the NRF measurements by an energy tunable LCS $\gamma$-ray source. In the present paper, we report the mechanism on how to determine hidden chemical compounds and a proof-of-principle experiment for this method. We measure an abundance ratio of ${ }^{14} \mathrm{C} /{ }^{14} \mathrm{~N}$ of a chemical

\footnotetext{
${ }^{a)}$ Electronic mail: hayakawa.takehito@jaea.go.jp.
}

material, melamine, shielded by thick iron and lead plates. Explosive materials include generally light elements such as carbon and nitrogen, and thus detection methods of the light elements under heavy shields is of importance in viewpoint of the secure society.

\section{PROPOSAL OF NONDESTRUCTIVE DETECTION METHOD OF A CHEMICAL COMPOUND AS MELAMINE}

Our proposed detection method is based on two key technologies: a NRF measurement technique and an energy tunable LCS $\gamma$-ray source. The NRF measurement provides a unique fingerprint of each nuclear species. If an incident $\gamma$-ray energy is identical with an excitation energy of a nucleus of interest, the incident $\gamma$-ray is effectively absorbed in the nucleus and subsequently the nucleus de-excite by emission of $\gamma$-rays. The energies of the states excited by NRF depend on the atomic number and mass of the nucleus of interest as shown in Fig. 1. By measuring the energies of the NRF scattering $\gamma$-rays, we can detect the nuclear species in a target material.

The energy tunable monochromatic $\gamma$-ray source enables us to perform such NRF measurement. The LCS $\gamma$-rays are generated by the inverse Compton scattering of laser photons with relativistic electrons. The maximum energy of the LCS $\gamma$-rays is determined by the energies of the electrons and the laser photons. In contrast, the lower limit of the energy width of the LCS $\gamma$-rays is determined by the scattering angle with a collimator. The energy of the scattered $\gamma$-ray $E_{\gamma}$ is presented by 


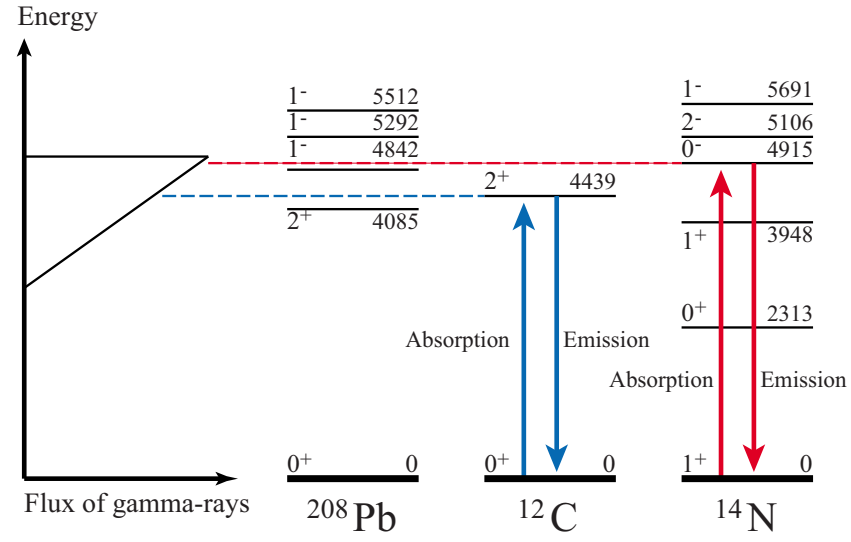

FIG. 1. (Color online) Schematic view of the nondestructive assay system based on NRF with the LCS $\gamma$-ray source. If the incident $\gamma$-ray energy is identical with the excitation energy of the nucleus of interest, the incident $\gamma$-ray is effectively absorbed in the nucleus and subsequently the nucleus de-excite by emission of the $\gamma$-ray.

$$
E_{\gamma}=\frac{E_{L}\left(1-\beta \cos \theta_{1}\right)}{1-\beta \cos \theta+\frac{E_{L}}{E_{e}}\left(1-\cos \theta_{2}\right)},
$$

where $E_{L}$ is the photon energy, $E_{e}$ is the electron energy, $\beta$ $=\sqrt{1-\gamma^{-2}}$ is the electron velocity in units of light speed $c$, and $\theta_{i}$ are scattering angles, as shown in Fig. 2. If we consider back-scattered photons from a head-on collision of the photons and the relativistic electrons: $\gamma \gg 1, \beta \simeq 1, \theta_{1} \simeq \theta_{2}$ $\simeq \pi$, and $\theta \ll 1$, we obtain the following equation:

$$
E_{\gamma}=\frac{4 \gamma^{2} E_{L}}{1+(\gamma \theta)^{2}+4 \gamma E_{L} /\left(m c^{2}\right)} .
$$

The energy of the scattering $\gamma$-ray decreases with increasing the scattering angle $\theta$. Therefore, by using a collimator to restrict the divergence of the $\gamma$-rays, we can limit the lowest energy of the LCS $\gamma$-rays and obtain a quasimonochromatic $\gamma$-ray beam.

Here we propose an extended nondestructive assay method for measuring a molecule or a chemical compound hidden by heavy shields such as iron plates of a thickness of several centimeters. The molecule or chemical compound consists of several elements and the elemental ratio depends on its chemical formula. By measuring the abundance ratio of key elements of the chemical compound of interest, we can detect it inside heavy shields. We can measure key elements at the same time with an energy tuned LCS $\gamma$-ray beam which energy width covers NRF $\gamma$-ray energies of

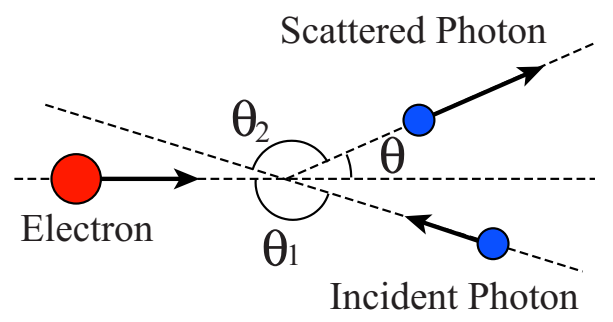

FIG. 2. (Color online) Schematic view of LCS. A high-energy photon $(\gamma$-ray) is generated via the Compton scattering of an incident photon with a relativistic electron. The energy of the scattering $\gamma$-ray depends on the scattering angle.

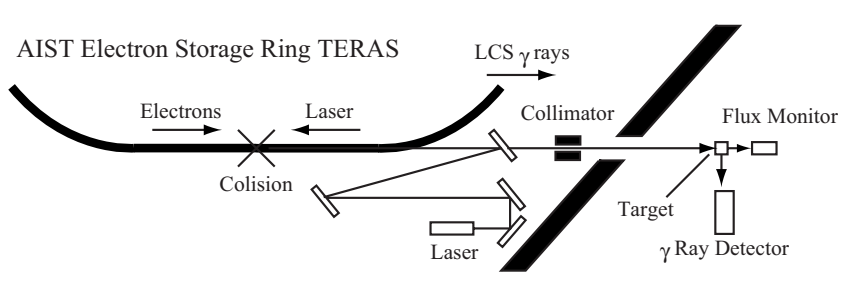

FIG. 3. Schematic view of experimental setup. The LCS $\gamma$-rays are generated by scattering of the laser photons with the electrons. The laser system is located inside the electron storage ring TERAS. The generated $\gamma$-rays are introduced into the experimental room.

nuclear species of the key elements. For example, the chemical formula of melamine is $\mathrm{C}_{3} \mathrm{H}_{6} \mathrm{~N}_{6}$ and the ratio of $(\mathrm{C} / \mathrm{N})_{\text {melamine }}$ is 0.5 . The natural isotopic abundances of ${ }^{12} \mathrm{C}$ and ${ }^{14} \mathrm{~N}$ are $98.89 \%$ and $99.634 \%$, respectively. Thus, the ratio ${ }^{12} \mathrm{C} /{ }^{14} \mathrm{~N}$ is nearly identical with the elemental ratio $\mathrm{C} / \mathrm{N}$. There are a $4439 \mathrm{keV}$ excited state in ${ }^{12} \mathrm{C}$ and a 4915 $\mathrm{keV}$ excited state in ${ }^{14} \mathrm{~N}$ (see Fig. 1). By using the LCS $\gamma$-ray beam with the energy width of 4.4-5.0 MeV, we can measure the abundance ratio of $\mathrm{C} / \mathrm{N}$ and identify the melamine.

\section{PROOF-OF-PRINCIPLE EXPERIMENT OF DETECTION OF MELAMINE BY USING NRF MEASUREMENT WITH A LCS $\gamma$-RAY SOURCE}

We perform a proof-of-principle experiment with detection of the melamine hidden behind heavy shields. We measure an abundance ratio ${ }^{12} \mathrm{C} /{ }^{14} \mathrm{~N}$ of the melamine hidden behind heavy shields. The experiment was carried out at a LCS $\gamma$-ray beam line of an electron storage ring TERAS in the National Institute of Advanced Industrial Science and Technology (AIST). ${ }^{13,15}$ A quasimonochromatic $\gamma$-ray beam was produced by head-on collisions of laser photons with a high-energy electron beam. We used an unpolarized $\mathrm{Nd}: \mathrm{YVO}_{4} Q$-switched laser with a wavelength of $1064 \mathrm{~nm}$, which was operated at a repetition rate of $20 \mathrm{kHz}$. The average power of the laser was $40 \mathrm{~W}$. The laser beam size at the collision point was $1.1 \mathrm{~mm}$ in diameter and the Rayleigh length was $21.2 \mathrm{~cm}$. A single laser pulse with a pulse length of $60 \mathrm{~ns}$ had a chance to collide with about 11 electron bunches in the collision region.

The electron beam energy was $530 \mathrm{MeV}$ and the maximum energy of the generated LCS $\gamma$-rays was approximately 5.0 MeV. The experimental setup is shown in Fig. 3. A lead collimator for limiting the energy spread of the LCS $\gamma$-rays located $6.2 \mathrm{~m}$ downstream from the collision point. The diameter of the collimator hole was $8 \mathrm{~mm}$ in order to obtain the $\gamma$-ray beam with the energy range of 4-5 MeV. The energy distribution of the $\gamma$-ray was measured by a High-Purity Germanium (HPGe) detector after NRF experiments and a detector response function calculated with the Monte Carlo code EGS4. The $\gamma$-ray flux throughout the NRF measurements was monitored by a large volume $\mathrm{NaI}(\mathrm{Tl})$ detector $\left(\phi 20 \times 30 \mathrm{~cm}^{2}\right)$ placed $2.2 \mathrm{~m}$ downstream from the target. The measured flux was up to $1 \times 10^{5}$ photons/s and the peak spectral density was up to 33 photons/s keV at $4915 \mathrm{keV}$. This flux was consistent with that estimated from the parameters of the laser and the electron beam. 


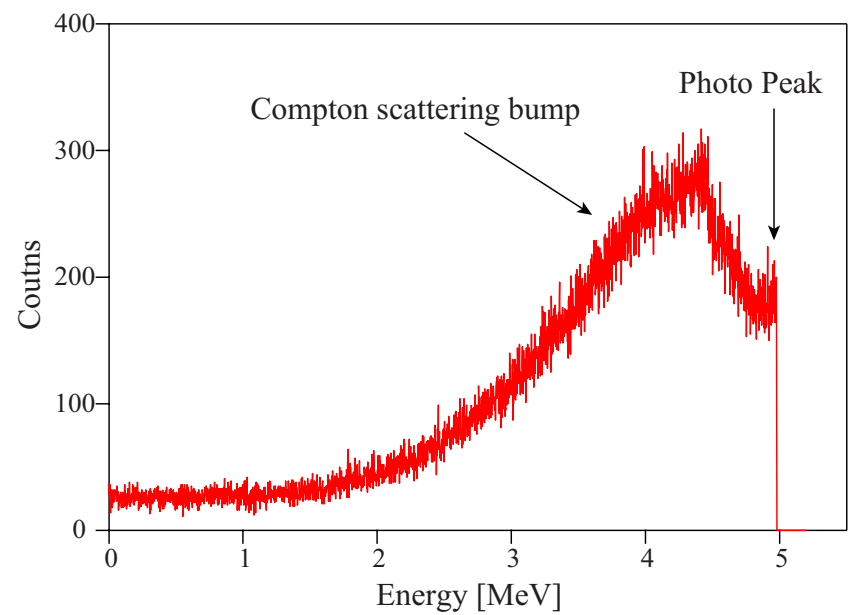

FIG. 4. (Color online) Measured energy spectrum of the incident LCS $\gamma$-rays. The $\gamma$-rays are measured by the HPGe detector. The HPGe detector cannot detect all the energy of the incident $\gamma$-ray of order of several MeV. The photo peak of the LCS $\gamma$-rays of $5 \mathrm{MeV}$ is observed but the bump is also observed.

The target is melamine powder inside a case of size of $\phi 32 \times 63 \mathrm{~mm}^{2}$ and the weight of the melamine powder is $35 \mathrm{~g}$. The distance from the collision point to the target was $10.3 \mathrm{~m}$. A $15-\mathrm{mm}$-thick iron plate and a 4-mm-thick lead plate are located in the front of the melamine target in order to simulate the situation of hidden materials. The melanin contains nuclear species of ${ }^{12} \mathrm{C}$ and ${ }^{14} \mathrm{~N}$. These nuclear species were populated by NRF with the incident LCS $\gamma$-rays and subsequently de-excited to the ground state by the emission of the $\gamma$-rays. These $\gamma$-rays were measured with a HPGe detector placed at an angle of $90^{\circ}$ with respect to the incident $\gamma$-ray beam. The efficiency of the HPGe detector was $120 \%$ relative to a $\phi 3 \times 3$ in. $^{2} \mathrm{NaI}$ detector. The signals of the HPGe detector were accumulated in coincidence with the laser pulse to reduce background noise originated from highenergy $\gamma$-rays generated by electron scattering with residual gases in the storage ring.

\section{RESULTS AND DISCUSSION}

Figure 4 shows an energy spectrum of the LCS $\gamma$-rays, which was measured by the HPGe detector. There is a photo peak and a bump generated by Compton scattering and $\gamma$-ray escape inside the HPGe detector. We obtain the energy spectrum of the incident LCS $\gamma$-rays using a response function of the HPGe detector calculated with the code EGS4. Figure 5 shows the deduced energy distribution of the incident LCS $\gamma$-ray beam. The deduced energy distribution shows typical features of the LCS $\gamma$-ray, i.e., a gradual increase in the $\gamma$-ray intensity at low energy and a steep drop at high energy. The maximum energy was approximately $5.0 \mathrm{MeV}$ and the energy spread was 20\% full width at half maximum. Figures 6 and 7 show a summed energy spectrum of the $\gamma$-rays emitted from the target for a period of $16 \mathrm{~h}$. We can see clear peaks at 4438 and $4915 \mathrm{keV}$, which are corresponding to NRF scattering $\gamma$-rays of ${ }^{12} \mathrm{C}$ and ${ }^{14} \mathrm{~N}$, respectively. The high $\mathrm{S} / \mathrm{N}$ ratio around the NRF peaks are one of excellent features of this type of measurement. We obtain a peak intensity ratio of $\left({ }^{12} \mathrm{C} /{ }^{14} \mathrm{~N}\right)_{\text {peak }}=1.0 \pm 0.18$ from the measured spectrum. Note

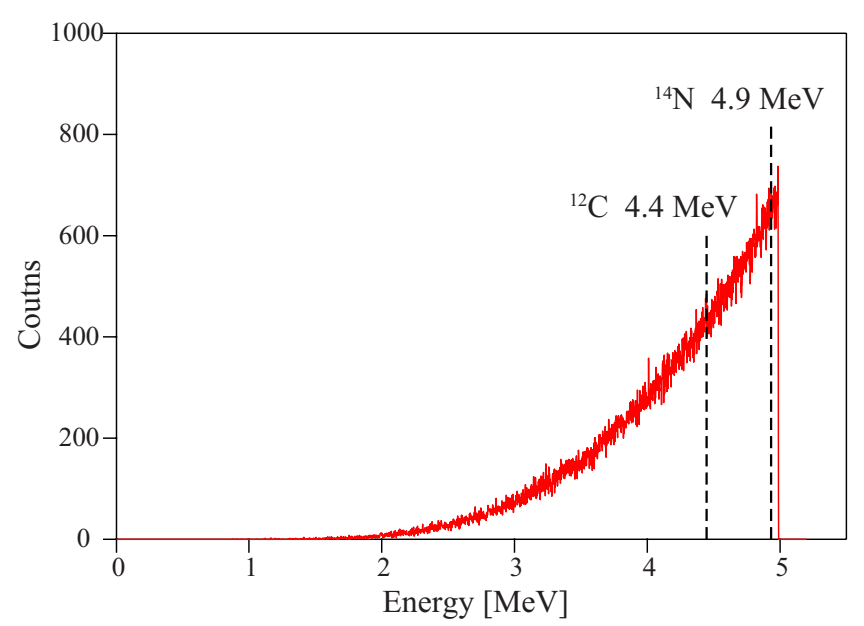

FIG. 5. (Color online) Calculated energy distribution of the incident LCS $\gamma$-rays. This is calculated from the measured spectrum and the detector response function evaluated by the Monte Carlo code EGS4. There is a sharp edge at the maximum energy. The energy spread is about $20 \%$.

that there is a peak at $4842 \mathrm{keV}$ in Fig. 7, which was originated from ${ }^{208} \mathrm{~Pb}$ inside the lead plate located on the front of the target. Since this $4842 \mathrm{keV}$ peak is completely isolated from both the photo peaks of ${ }^{12} \mathrm{C}$ and ${ }^{14} \mathrm{~N}$, there is no effect on the present analysis.

Here we evaluated an experimental ratio $\left({ }^{12} \mathrm{C} /{ }^{14} \mathrm{~N}\right)_{\exp }$ in order to compare with the exact value of $(\mathrm{C} / \mathrm{N})_{\text {melamine }}=0.5$. The scattering cross-section over one level is given by

$$
\begin{aligned}
& \frac{d \sigma}{d \Omega}=g\left(\frac{\pi \hbar c}{E_{\gamma}}\right)^{2} \frac{\Gamma_{0} \Gamma_{i}}{\Gamma} \frac{W(\theta, \phi)}{4 \pi}, \\
& g=\frac{2 J_{1}+1}{2 J_{0}+1},
\end{aligned}
$$

where $E_{\gamma}$ is the $\gamma$-ray energy, $g$ is the spin factor, $J_{0}$ and $J_{1}$ are the spin of the ground state and the excited state, respectively, $\Gamma, \Gamma_{0}$, and $\Gamma_{i}$ are the total width, the ground state decay width, and the partial decay width, respectively, and

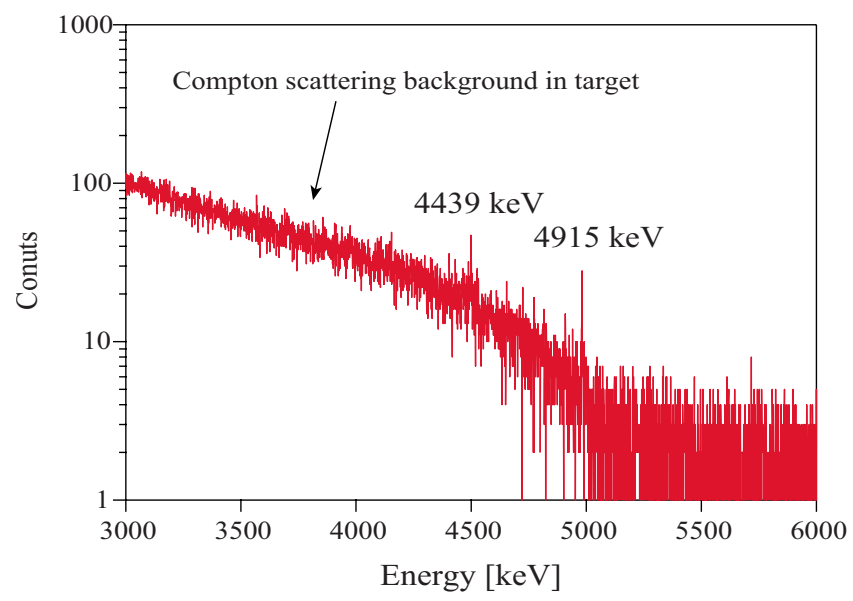

FIG. 6. (Color online) Measured NRF $\gamma$-rays from the melamine target. The maximum energy of the incident LCS $\gamma$-rays is $5.0 \mathrm{MeV}$ and thus the background originated from the Compton scattering in the target is observed at the energy region lower than $5.0 \mathrm{MeV}$. The background increases with decreasing the $\gamma$-ray energy. There are clear photo peaks at 4915 and $4439 \mathrm{keV}$ with high $\mathrm{S} / \mathrm{N}$ ratios. 


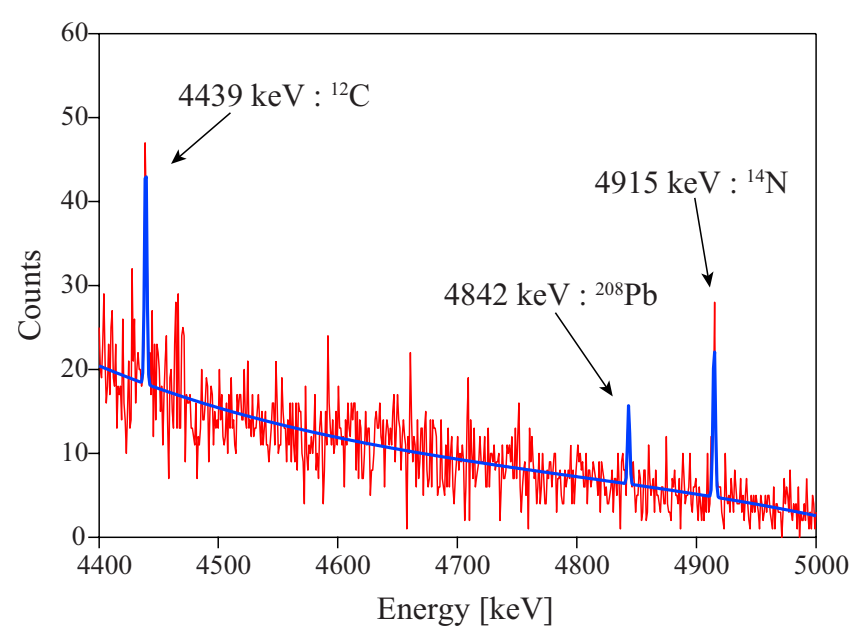

FIG. 7. (Color online) Measured NRF $\gamma$-rays from the melamine target. There are clear photo peaks at 4915 and $4439 \mathrm{keV}$, which are corresponding to the NRF scattering $\gamma$-rays of ${ }^{12} \mathrm{C}$ and ${ }^{14} \mathrm{~N}$. In addition, the $\gamma$-ray of ${ }^{208} \mathrm{~Pb}$ inside the lead shield in the front of the target is also observed.

$W(\theta)$ is the normalized angular distribution. The angular distribution of the NRF scattering $\gamma$-rays through dipole transitions in a case of ${ }^{14} \mathrm{~N}$ is given by

$$
W_{d}(\theta)=\frac{3}{4}\left(1+\cos ^{2} \theta\right) \text {, }
$$

and through quadrupole transitions in a case of ${ }^{12} \mathrm{C}$ by

$$
W_{q}(\theta)=\frac{5}{4}\left(1-3 \cos ^{2} \theta+4 \cos ^{4} \theta\right) .
$$

The cross-section is determined by the spin factor, the $\gamma$-ray energy, and the energy width. The experimental ratio $\left({ }^{12} \mathrm{C} /{ }^{14} \mathrm{~N}\right)_{\exp }$ can be obtained by

$$
\begin{aligned}
\left({ }^{12} \mathrm{C} /{ }^{14} \mathrm{~N}\right)_{\exp }= & \frac{g\left({ }^{14} \mathrm{~N}\right)}{g\left({ }^{12} \mathrm{C}\right)} \frac{\Gamma_{0}\left({ }^{14} \mathrm{~N}\right)}{\Gamma_{0}\left({ }^{12} \mathrm{C}\right)} \frac{W_{d}}{W_{q}} \frac{\epsilon(4915 \mathrm{keV})}{\epsilon(4439 \mathrm{keV})} \\
& \times \frac{I(4915 \mathrm{keV})}{I(4439 \mathrm{keV})} \frac{E_{\gamma}\left({ }^{14} \mathrm{~N}\right)^{2}}{E_{\gamma}\left({ }^{12} \mathrm{C}\right)^{2}}\left({ }^{12} \mathrm{C} /{ }^{14} \mathrm{~N}\right)_{\text {peak }},
\end{aligned}
$$

where $\epsilon(x)$ is the photo peak efficiency at the energy of $x$, $I(x)$ is the relative intensity of the energy distribution of the incident LCS $\gamma$-rays at the energy of $x$.

The spin factors are 5 and $1 / 3$ for ${ }^{12} \mathrm{C}\left(2^{+} \rightarrow 0^{+}\right)$and ${ }^{14} \mathrm{~N}\left(0^{+} \rightarrow 1^{+}\right)$, respectively. The ground state decay width $\Gamma_{0}$ are $10.8 \pm 0.06 \mathrm{meV}$ and $84 \pm 16 \mathrm{meV}$ for ${ }^{12} \mathrm{C}$ and ${ }^{14} \mathrm{~N}$, respectively. The decay width of ${ }^{14} \mathrm{~N}$ is obtained from the measured half-life of its excited state. The ratio of $W_{d} / W_{q}$ is approximately 0.6. The detection efficiency of the HPGe detector is calculated with the code EGS4. The ratio $\epsilon(4915 \mathrm{keV}) / \epsilon(4439 \mathrm{keV})$ is almost 1.0 since the efficiency is not sensitive to the $\gamma$-ray energy in this energy region. The intensities at the two energies are obtained from the measured energy distribution of the incident LCS $\gamma$-rays, as shown in Fig. 5. The intensity ratio of $I(4439 \mathrm{keV}) / I(4915 \mathrm{keV})$ is 0.68 . We finally obtain the experimental ratio $\left({ }^{12} \mathrm{C} /{ }^{14} \mathrm{~N}\right)_{\exp }=0.39 \pm 0.12$. which is consistent with the melamine ratio of $\left({ }^{12} \mathrm{C} /{ }^{14} \mathrm{~N}\right)_{\text {melamine }}=0.5$. The experimental uncertainty dominantly consists of the statistical error and the half-life uncertainty of the $4915 \mathrm{keV}$ state in
${ }^{14} \mathrm{~N}$. The present result suggests that our proposed method enables us to detect chemical compounds as well as elemental composition of a hidden material.

For the nondestructive detection of materials in an industrial scale, we have designed a high-flux $\gamma$-ray facility utilizing an energy-recovery linac (ERL) equipped with a superconducting accelerator. ${ }^{16}$ As an example, we take a high-flux $\gamma$-ray source based on a $350 \mathrm{MeV}$ ERL. High-flux $\gamma$-ray beams of energy between $0.5-9 \mathrm{MeV}$ are generated from the Compton scattering with a Yb-doped fiber laser. We can obtain a total $\gamma$-ray flux of $1.0 \times 10^{13}$ photons/s and a peak spectral density of $7.0 \times 10^{9}$ photons/s keV. With such high-flux LCS $\gamma$-ray source, we can measure the $1 \mathrm{~g}$ melamine with measurement time of only $1 \mathrm{~s}$ within the uncertainty of $20 \%$. This uncertainty dominantly originates from the half-life uncertainty of the $4915 \mathrm{keV}$ excited state in ${ }^{14} \mathrm{~N}$ and its precise measurement is highly desired.

\section{SUMMARY}

We propose an assay method for measuring molecules and chemical compounds hidden by heavy shields such as iron of the thickness of several centimeters. Our proposed method is based on a NRF measurement technique and energy tunable LCS $\gamma$-rays. We perform a proof-of-principle experiment to detect a shielded chemical compound of melamine, $\mathrm{C}_{3} \mathrm{H}_{6} \mathrm{~N}_{6}$. We detect an abundance ratio of ${ }^{12} \mathrm{C} /{ }^{14} \mathrm{~N}$ in the same NRF measurement with the LCS $\gamma$-rays provided from an electron storage ring TERAS. The observed ratio is identical with the exact ratio within the experimental uncertainty of $31 \%$. The present result suggests that our proposed method with a high-flux $\gamma$-ray source will enable us to detect molecules and chemical compounds hidden by shields with short time. Explosive materials include generally the carbon and nitrogen, and thus the present proposed method is of importance in viewpoint of the secure society.

\section{ACKNOWLEDGMENTS}

This work has been supported in part by Grants-in-Aid for Scientific Research in Japan (Grant Nos. 19540289, 20612014, and 18340071).

${ }^{1}$ T. Sakurai, K. Kosako, and T. Mori, Proceedings of the Third Meeting on Monte Carlo Simulation Code, 2004 (unpublished) p. 168.

${ }^{2}$ W. Bertozzi and R. J. Ledoux, Nucl. Instrum. Methods Phys. Res. B 241, 820 (2005).

${ }^{3}$ D. A. Close, L. A. Franks, and S. M. Kocimski, Nucl. Instrum. Methods Phys. Res. A 220, 531 (1984).

${ }^{4}$ M. Haruyama, M. Takase, and H. Tobita, J. Nucl. Sci. Technol. 45, 432 (2008).

${ }^{5}$ J. Pruet, D. P. McNabb, C. A. Hagmann, F. V. Hartemann, and C. P. J. Barty, J. Appl. Phys. 99, 123102 (2006).

${ }^{6}$ V. N. Litvinenko, B. Burnham, M. Emamian, N. Hower, J. M. J. Madey, P. Morcombe, P. G. O'Shea, S. H. Park, R. Sachtschale, K. D. Straub, G. Swift, P. Wang, Y. Wu, R. S. Canon, C. R. Howell, N. R. Roberson, E. C. Schreiber, M. Spraker, W. Tornow, H. R. Weller, I. V. Pinayev, N. G. Gavrilov, M. G. Fedotov, G. N. Kulipanov, G. Y. Kurkin, S. F. Mikhailov, V. M. Popik, A. N. Skrinsky, N. A. Vinokurov, B. E. Norum, A. Lumpkin, and B. Yang, Phys. Rev. Lett. 78, 4569 (1997).

${ }^{7}$ F. V. Hartemann, W. J. Brown, D. J. Gibson, S. G. Anderson, A. M. Tremaine, P. T. Springer, A. J. Wootton, E. P. Hartouni, and C. P. Barty, Phys. Rev. ST Accel. Beams 8, 100702 (2005). 
${ }^{8}$ K. Kawase, M. Kando, T. Hayakawa, I. Daito, S. Kondo, T. Homma, T. Kameshima, H. Kotaki, L.-M. Chen, Y. Fukuda, A. Faenov, T. Shizuma, M. Fujiwara, S. V. Bulanov, T. Kimura, and T. Tajima, Rev. Sci. Instrum. 79, 053302 (2008).

${ }^{9}$ G. Priebe, D. Laundy, M. A. Macdonald, G. P. Diakun, S. P. Jamison, L. B. Jones, D. J. Holder, S. L. Smith, P. J. Phillips, B. D. Fell, B. Sheehy, N. Naumova, I. V. Sokolov, S. Ter-Avetisyan, K. Spohr, G. A. Krafft, J. B. Rosenzweig, U. Schramm, F. Grüner, G. J. Hirst, J. Collier, S. Chattopadhyay, and E. A. Seddon, Laser Part. Beams 26, 649 (2008).

${ }^{10}$ T. Hayakawa, S. Miyamoto, Y. Hayashi, K. Kawase, K. Horikawa, S. Chiba, K. Nakanishi, H. Hashimoto, T. Ohta, M. Kando, T. Mochizuki, T. Kajino, and M. Fujiwara, Phys. Rev. C 74, 065802 (2006).

${ }^{11}$ T. Shizuma, T. Hayakawa, H. Ohgaki, H. Toyokawa, T. Komatsubara, N.
Kikuzawa, A. Tamii, and H. Nakada, Phys. Rev. C 78, 061303(R) (2008).

${ }^{12}$ H. Toyokawa, H. Ohgaki, T. Mikado, and K. Yamada, Rev. Sci. Instrum. 73, 3358 (2002)

${ }^{13}$ H. Ohgaki, S. Sugiyama, T. Yamazaki, T. Mikado, M. Chiwaki, K. Yamada, R. Suzuki, T. Noguchi, and T. Tomimasu, IEEE Trans. Nucl. Sci. 38, 386 (1991).

${ }^{14}$ R. Hajima, T. Hayakawa, N. Kikuzawa, and E. Minehara, J. Nucl. Sci. Technol. 45, 441 (2008).

${ }^{15}$ H. Ohgaki, H. Toyokawa, K. Kudo, N. Takeda, and T. Yamazaki, Nucl. Instrum. Methods Phys. Res. A 455, 54 (2000).

${ }^{16}$ R. Hajima, N. Kikuzawa, T. Hayakawa, and E. Minehara, Proceedings of the Eigth International Topical Meeting on Nuclear Applications and Utilization of Accelerators, 2007 (unpublished), p. 182. 